\title{
APLICACIONES CARTOGRÁFICAS PARA LA VALORACIÓN DE SUPERFICIES DE ESCORRENTÍA. RIU GIRONA (ALICANTE) ${ }^{1}$
}

\author{
Marcos Moll Barber ${ }^{2}$ \\ Máster en Planificación y Gestión de Riesgos Naturales \\ por el Instituto Interuniversitario de Geografia \\ Universidad de Alicante
}

\section{RESUMEN}

En el presente artículo se plantea una metodología centrada en elaborar una cartografía que sirva para evaluar las diferentes superficies de escorrentía que se encuentran en una cuenca. El marco geográfico sobre el cual se aplica a modo de experimento es el río Girona, localizado en el norte de la provincia de Alacant. Para llevar a cabo la metodología ha sido imprescindible revisar investigaciones sobre coeficiente de escorrentía para llegar a confeccionar una fórmula matemática que ayudara a obtenerlo. Para ello se ha trabajado con distintas variables espaciales integradas en un Sistema de Información Geográfica, con tal de realizar combinaciones entre pendientes, litología y usos del suelo y cubierta vegetal. Dentro de la metodología seguida, el proceso de fotointerpretación ha jugado un papel importantísimo como método de adaptación a la escala de trabajo y como herramienta de evaluación de la superficie.

Palabras clave: fotointerpretación, coeficiente de escorrentía, sistemas de información geográfica, río Girona.

\section{ABSTRACT}

\section{Cartographic tools to evaluate surface runoffs. Girona river (Alicante)}

This article describes the methodology used in the cartography, which is used to evaluate the different surface runoffs in the basin. The geographic focus of this experiment is the Girona river, located in the north of Alacant Province. It was necessary to review research studies on runoff coefficients in order to identify the appropriate methodology for this study. The methodology selected helped us to choose a mathematical formula that was constructive in finding the coefficient. We have worked with different spatial variables integrated into a Geographical Information System, in order to combine slops, lithology, land uses and vegetation. In the methodology followed, the process of photo-interpretation was crucial for adapting to the scale of the project and as an assessment tool of the surface.

Key words: photo-interpretation, runoff coefficient, geographic information system, Girona river.

\section{CONSIDERACIONES PRELIMINARES SOBRE ESCORRENTÍA}

El estudio que aquí se plantea, centrado en evaluar el estado de las superficies de escorrentía, se trata de una aportación metodológica que pretende ser genérica y, además, abierta a modificaciones, con el propósito de perfeccionar el método y aplicarlo a otras cuencas, he aquí la importancia del mismo. En todo momento las particularidades locales han ejercido, y de hecho ejercen, un papel fundamental a la hora de confeccionar el método cartográfico que se propone.

\footnotetext{
1 El artículo deriva del Trabajo Final del Máster en Planificación y Gestión de Riesgos Naturales impartido en el Instituto Interuniversitario de Geografía de la Universitat d'Alacant y fue dirigido por el Dr. D. Pablo Giménez Font.

2 korkus100@hotmail.com
} 
Entendiendo la escorrentía como la parte del agua precipitada que no se evapora, no se evapotranspira ni se intercepta, ni se almacena ni se infiltra; el coeficiente de escorrentía $\left(C_{e}\right)$ es la porción de agua que circula en superficie en relación al agua total precipitada, expresándose en tanto por uno. Éste es fundamental y muy útil para calcular el caudal de una cuenca y, de este modo, poder adaptar cualquier obra de ingeniería al caudal esperado, o bien, para tomar medidas en Ordenación y Planificación del Territorio que ayuden a mitigar el riesgo de inundación.

A lo largo del siglo XX en el ámbito científico han aparecido diferentes métodos para extraer el coeficiente de escorrentía. Muestra de ello son, Horton (1933), Musgrave (1955), Molchanov (1960), Marsh (1978), Benítez, et al., (1980), Prevert (1998), etc. Todos ellos cuantifican el coeficiente de escorrentía en diferentes superficies teniendo en cuenta parámetros como el grado de pendiente, el uso del suelo y las litofacies y formaciones superficiales.

Marsh (1978) examina coeficientes de escorrentía de espacios rurales y urbanos, y hace constancia que los primeros presentan un $C_{e}$ menor. Además de ello, cabe subrayar que en las áreas rurales Marsh establece tres tipos de suelo (franco-arenoso; franco-limoso y franco-arcilloso; y arcillosos) y tres grados de pendiente, uno llano, de 0 al 5\%; otro ondulado, de 5 al 10\%; y por último, accidentado, entre 10 y $30 \%$. De los valores tomados cabe indicar que en los espacios urbanos no se consideran las variaciones de pendiente.

El equipo de Benítez et al., (1980) amplía los rangos de pendiente en las muestras que se toman de $C_{e}$, pasando de tres a cinco grupos, entre 0 y $1 \%$, entre 1 y $5 \%$, entre 5 y $20 \%$, entre 20 y $50 \%$ y superiores al 50\%. Además en éste, directamente se clasifican los suelos atendiendo a la capacidad de infiltración en permeables, semipermeables e impermeables.

En cuanto al método de Prevert (1998), citado en Ibáñez et al., (2007), clasifica el suelo según su textura en gruesa (arenoso-limoso y limoso-arenoso), mediana (limoso y limoso-arcilloso) y fina (arcillas). Por lo que se refiere a la pendiente, este método distingue cuatro grupos, entre 0 y $5 \%$ de pendiente, entre 5 y $10 \%$, entre 10 y $30 \%$ y mayores del $30 \%$.

Cabe indicar que el fenómeno de infiltración que se produce en las litofacies y formaciones superficiales también ha sido muy estudiado, ejemplo de ello son Kostiakov (1932), Horton (1933), Philip (1957), Musgrave et al., (1964) y el Soil Conservation Service (1972), entre otros. A grandes rasgos, el grado de infiltración viene determinado por la relación entre poros, estructura y estratificación, tamaño de las partículas, fisuras, temperatura del agua y por el aire estancado en su interior (Anglone, et al., 2006) y, además, también está determinado por los desniveles topográficos de la cuenca (Gil, et al., 1986) y por las condiciones de humedad en que se encuentra el suelo (Smith, 1972).

De todos los métodos comentados y en lo que aquí interesa, cabe destacar los siguientes aspectos: que a medida que aumenta la pendiente también lo hace la escorrentía; que cuanta mayor sea la densidad de vegetación, menor será la escorrentía; que las áreas urbanas generan más escorrentía que las rurales; y que a menor permeabilidad de las litofacies y formaciones superficiales más escorrentía se producirá.

\section{OBJETIVOS, METODOLOGÍA Y TÉCNICAS PARA VALORAR SUPERFICIES DE ESCORRENTÍA}

La cuenca del río Girona queda representada en las Hojas 796 (Gandia), 821 (Alcoi) y 822 (Benissa) del Mapa Topográfico Nacional a escala 1:50.000. Se trata de una pequeña cuenca hidrográfica del Mediterráneo occidental que presenta un régimen irregular, fuertes desniveles topográficos y relieves montañosos cercanos al mar.

Entre el 11 y el 12 de octubre del 2007 tuvo lugar un episodio de lluvias torrenciales con una elevada intensidad horaria que causó daños en infraestructuras y bienes. Muchas calles de los pueblos de Beniarbeig, El Verger y Els Poblets, situados en abanicos aluviales de las partes bajas de la cuenca, quedaron inundados. Este episodio ha sido estudiado por Francesca Segura (2009), quien, con la ayuda de los SIG, puso énfasis en aspectos geomorfológicos (abanicos aluviales, paleocauces, etc.) y en la alteración antrópica acontecida en el espacio inundable de la cuenca. Por esa razón, en la metodología 
que aquí se plantea, se tendrán en cuenta que las superficies de escorrentía han mutado debido a cambios de uso del suelo, como por ejemplo abandonos de cultivos, aumento del espacio urbanizado, abandono del aprovechamiento forestal, incendios forestales pasados, etc.

Los objetivos que se persiguen en este artículo se centran en valorar la superficie de escorrentía de la cuenca del río Girona con el fin de plantear un procedimiento cartográfico que sirva de ayuda a futuras investigaciones que pretendan gestionar y planificar el riesgo de inundación en cualquier cuenca hidrográfica, ya sea perfilando este modelo o usándolo como tal. Para conseguir tales objetivos es necesario considerar en el SIG las diferentes variables espaciales que repercuten en la escorrentía. En la siguiente figura se jerarquiza el proceso cartográfico aplicado.

Figura 1. Esquema metodológico aplicado a la cuenca del Riu Girona.

\section{PROCEDIMIENTO CARTOGRÁFICO}

FASE PREVIA: Selección de la información, software y confección del área de estudio

- Recopilación de bases de datos (CRS: ETRS89): SIOSE (IGN), MDT05 (IGN), Ortofoto 2005 (ICV), límites de la cuenca del Girona y de la Confederación Hidrográfica del Júcar (CHJ).

- Programas SIG: SEXTANTE, GVSIG y QGIS.

- Confección del área de estudio: modificación y adaptación de los límites de cuenca a la escala 1:5.000.

FASE INICIAL: Confección de las capas de información geográfica

- Usos del suelo y vegetación: fotointerpretación (Ortofoto 2005) y modificación del SIOSE, corrección de errores, consideración de ejes de comunicación interiores e introducción de nuevas terminologías (afines al estudio).

- Pendientes: a partir del MDT05 se aplica el algoritmo de ajuste polinómico de segundo grado de Zevenbergen \& Thorne et al., 1987 y se reclasifica en tres clases ( $0-5 \% ; 5-10 \% ;>10 \%)$.

- Litofacies y formaciones superficiales: fotointerpretación de la materiales según la permeabilidad y adaptación escalar.

\section{FASE DE VALORACIÓN}

- Confección del coeficiente para valorar las superficies de escorrentía.

- Revisión de los coeficientes de escorrentía: Marsh (1978), Prevert (1998), etc. y cuantificaciones de incrementos al cambiar la permeabilidad y la pendiente para valorar la información contenida en las capas.

- Capa final (geoproceso de intersección, suma de valores y división): valoración de la superficie de escorrentía.

\section{FASE DE VERIFICACIÓN}

- Comprobar el resultado y ver si concuerda con los coeficientes de escorrentía.

- Salidas de campo para contrastar los resultados con la realidad.

- Modificación: en caso de obtener resultados no esperados, replantear el coeficiente y las valoraciones.

El eje fundamental de trabajo gira entorno a una mejora de la cartografía disponible y al proceso de fotointerpretación de la superficie de la cuenca en el año 2005, dos años antes de la riada anteriormente comentada. La fotointerpretación ha sido de marcada utilidad en la tarea de examinar y evaluar los usos del suelo y la cubierta vegetal, así como las litofacies y formaciones superficiales de la cuenca.

\subsection{Fase cartográfica previa: programas, escala, bases de datos, CRS y límites}

Para poder realizar el estudio se han utilizado programas de libre distribución y de código abierto vinculados a los SIG, éstos han sido GVSIG, SEXTANTE y QGIS.

La escala de trabajo que se elige es de 1:5.000 porque la computadora que se utiliza tiene una RAM de $2 \mathrm{~Gb}$ y en caso de usar una escala de mayor detalle dificultaría el proceso de elaboración cartográfica.

El Sistema de Coordenadas de Referencia (CRS) que se utiliza es el European Terrestrial System 1989 (ETRS89) que trabaja en coordenadas UTM (Universal Transverse Mercator) y que fue aprobado en España mediante el Real Decreto 1071/2007, de 27 de julio, como adaptación a las normativas europeas, dejando atrás el European Datum 1950 (ED50).

Las bases de datos geográficos que se necesitan en esta aportación son: los límites de la cuenca extraído de la Confederación Hidrográfica del Júcar (CHJ) a escala 1:1.000.000 - 1:500.000; el Sistema de Información sobre Ocupación del Suelo en España (SIOSE) del año 2005 a escala 1:25.000; el Modelo 
Digital del Terreno (MDT) con un píxel de $5 \mathrm{~m}$ del Instituto Geográfico Nacional (IGN); la Ortofoto del vuelo de 2005 del Institut Cartogràfic Valencià (ICV) con un píxel de medio metro; y, las zonas urbanas, las vías de comunicación y las curvas de nivel del ICV a escala 1:5.000.

Una vez descargada la información necesaria, se pasa a modificar a manualmente el límite de la cuenca para adaptarlo a la escala de trabajo usando como guía el MDT05 y las curvas de nivel del ICV a escala 1:5.000. Mediante este procedimiento se amplía la zona costera y se perfilan mejor las divisorias de aguas. Según la Confederación Hidrográfica del Júcar la cuenca ocupa una superficie de 111,37 km² y, una vez modificada, el área de la cuenca pasa a ocupar una superficie mayor, de $117,36 \mathrm{~km}^{2}$.

\subsection{Fase cartográfica inicial: confección de las capas de información geográfica}

\subsubsection{Los usos del suelo y cubierta vegetal}

Para confeccionar la capa de usos del suelo y cubierta vegetal, se ha usado la base de datos del SIOSE. En primer lugar, utilizando como límite el área de estudio modificado se extrae la información de la cuenca mediante el geoproceso de cortar. Posteriormente se descifran los códigos empleados en la capa. Seguidamente se contrastan y modifican los polígonos del SIOSE usando como guía la Ortofoto del año 2005 del ICV para ir adaptando el contenido a la escala de trabajo. Para llevar a cabo este procedimiento de fotointerpretación, las salidas de campo han jugado un papel importante.

Figura 2. Ejemplo de masa forestal desarrollada sobre antiguas terrazas de cultivo, La Vall de Laguar (2010).

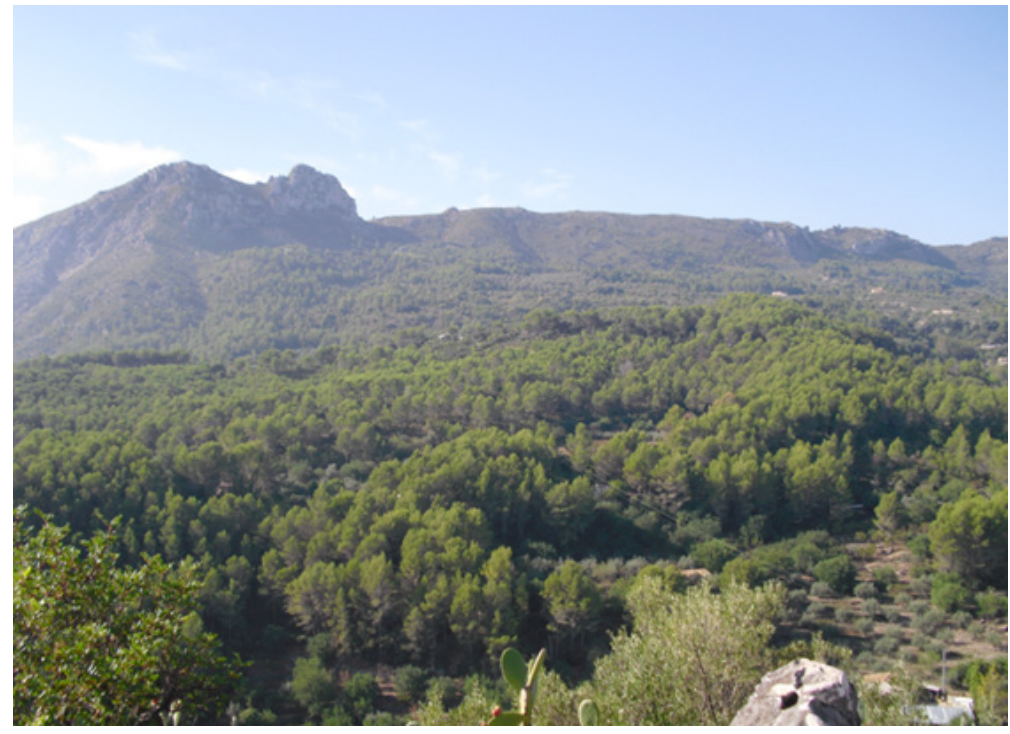

Elaboración própia.

Una vez fotointerpretados los usos del suelo y la cubierta vegetal en el año 2005, se comprueba la validez de las geometrías usando la versión 1.7.4. de QGIS, habiendo resultado más de 160 nodos conflictivos, que posteriormente se corrigen. Seguidamente se consideran las carreteras de interior utilizando la información vectorial del ICV a escala 1:5.000. A las carreteras en formato de líneas se les aplica un área de influencia de $2,5 \mathrm{~m}$ a cada lado para posteriormente integrarlas en la capa de usos del suelo y vegetación, ya que en la capa del SIOSE no aparecían dichas carreteras interiores. Finalmente se opta por sustituir las denominaciones por otras afines a los propósitos de estudio (figura 3).

En la fotointerpretación se han tenido en cuenta las terrazas abandonadas porque dicha situación implica cambios en la infiltración y el discurrir de las aguas superficiales (Cerdà, 1994). También se consideran las situaciones post-incendio por el mismo hecho de mutar el escenario de escorrentía (Úbeda 2000; Cerdà et al., 2006; Llovet et al., 2011), que en la zona de estudio se corresponden con los afloramientos rocosos localizados en las zonas montañosas, aunque a grandes rasgos estos espacios se están regenerado favorablemente. 
Figura 3. Introducción de nuevas terminologías en la capa de usos del suelo y coberturas vegetales y características.

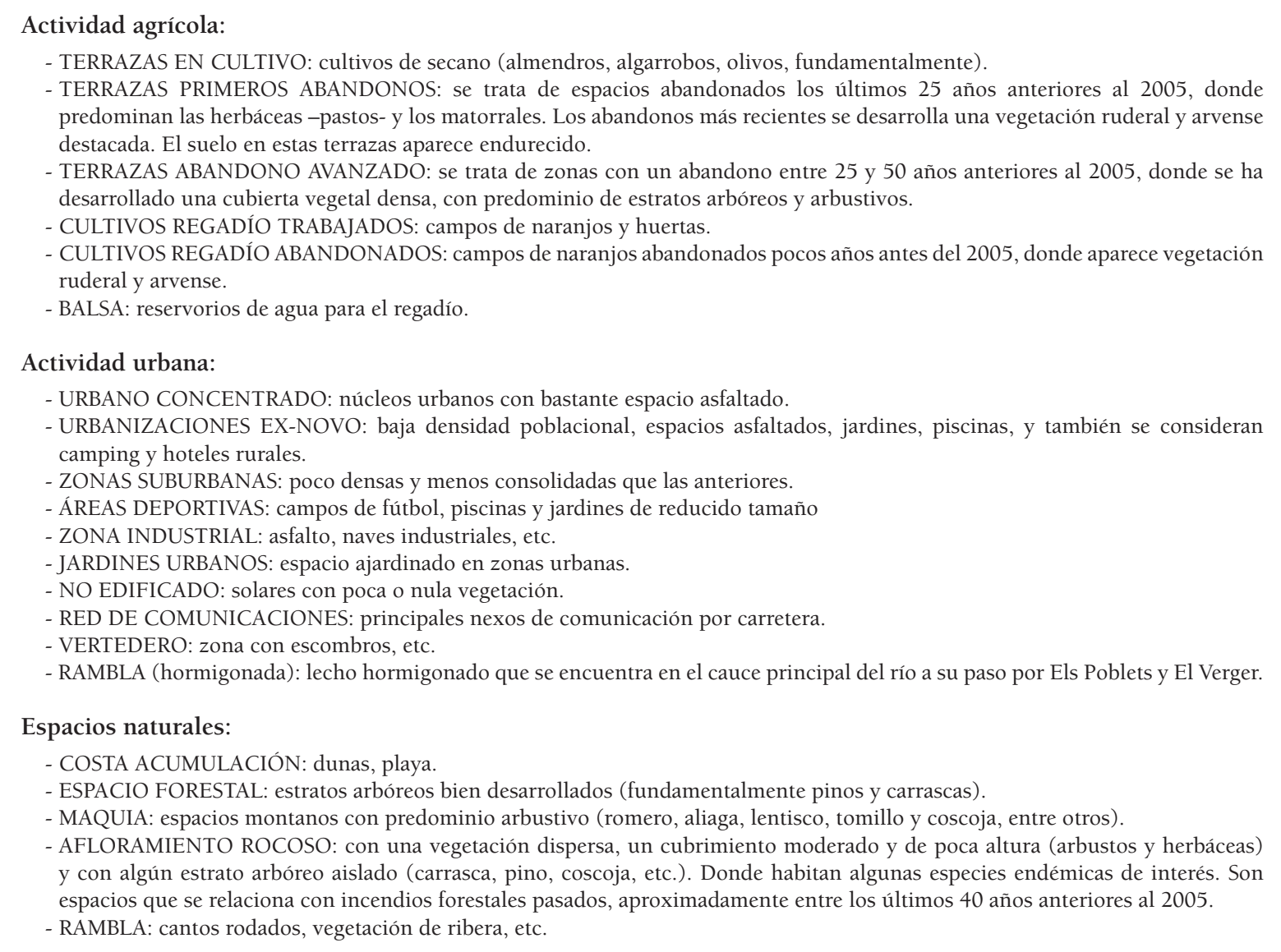

\subsubsection{Las pendientes}

Para obtener la capa de pendientes se utiliza el Modelo Digital del Terreno con un píxel de cinco metros (MDT5). En primer lugar se rellenan las celdas sin datos del MDT5, para no arrastrar errores en el proceso de confección de la capa de pendientes en caso de existencia de celdas sin datos. A partir del MDT05 resultante se le aplica el algoritmo de ajuste polinómico de segundo grado de Zevenbergen \& Thorne et al., 1987 y se reclasifica el resultado en tres grandes grupos de pendientes, entre 0 y $5 \%$, entre 5 y $10 \%$ y superiores al $10 \%$. Posteriormente se ajusta mediante la función cortar usando el área de la cuenca en formato ascii, el resultado del cual se vectoriza.

\subsubsection{Las litofacies y formaciones superficiales}

En cuanto a la elaboración de esta capa, se ha optado por digitalizar la información a partir de una fotointerpretación, porque no hay fuentes disponibles a escala 1:5.000 de litofacies y formaciones superficiales. Para ello se ha usado la Ortofoto del 2005 del ICV. Hay que advertir que en caso de emplear otras escalas se derivarían en errores geométricos en la combinación final de las variables consideradas en la valoración.

En este proceso de fotointerpretación, se ha pasado directamente a digitalizar las superficies según su grado de permeabilidad en: muy permeables, permeables y semipermeables. Se han considerado muy permeables las zonas con afloramientos calcáreos localizados en zonas montañosas por su elevado grado de carstificación (fisuras, cavernas, cañones, etc.), así como los suelos compuestos por gravas y arenas; permeables los suelos compuestos por arenas finas, limos y margas que se encuentran en taludes, glacis y conos de deyección y, además, el lecho del río junto a sus márgenes; y semipermeables los suelos con presencia de arcillas, limos, margas y gravas, éstos, ubicados en los abanicos aluviales y en la llanura de inundación. 


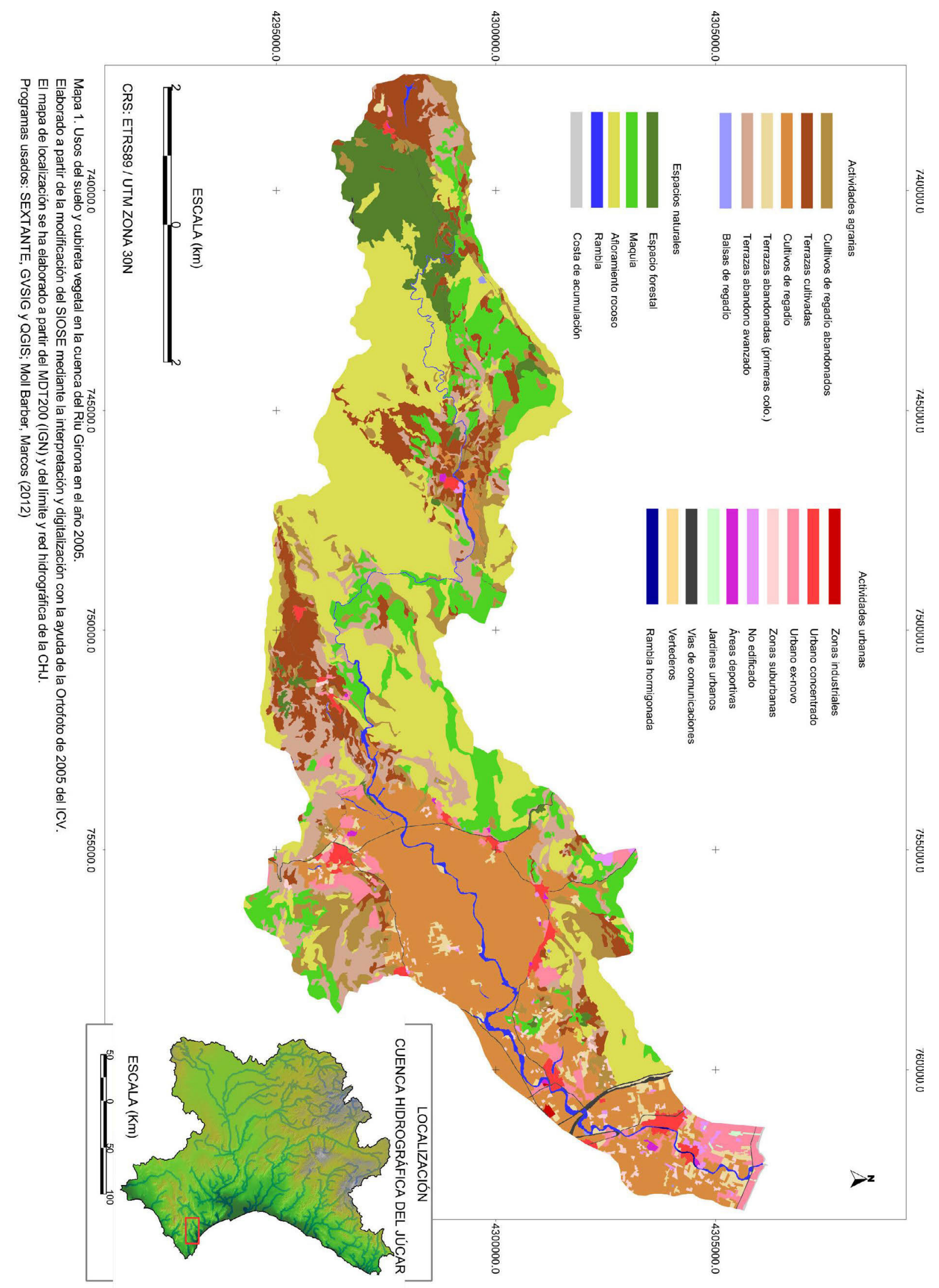




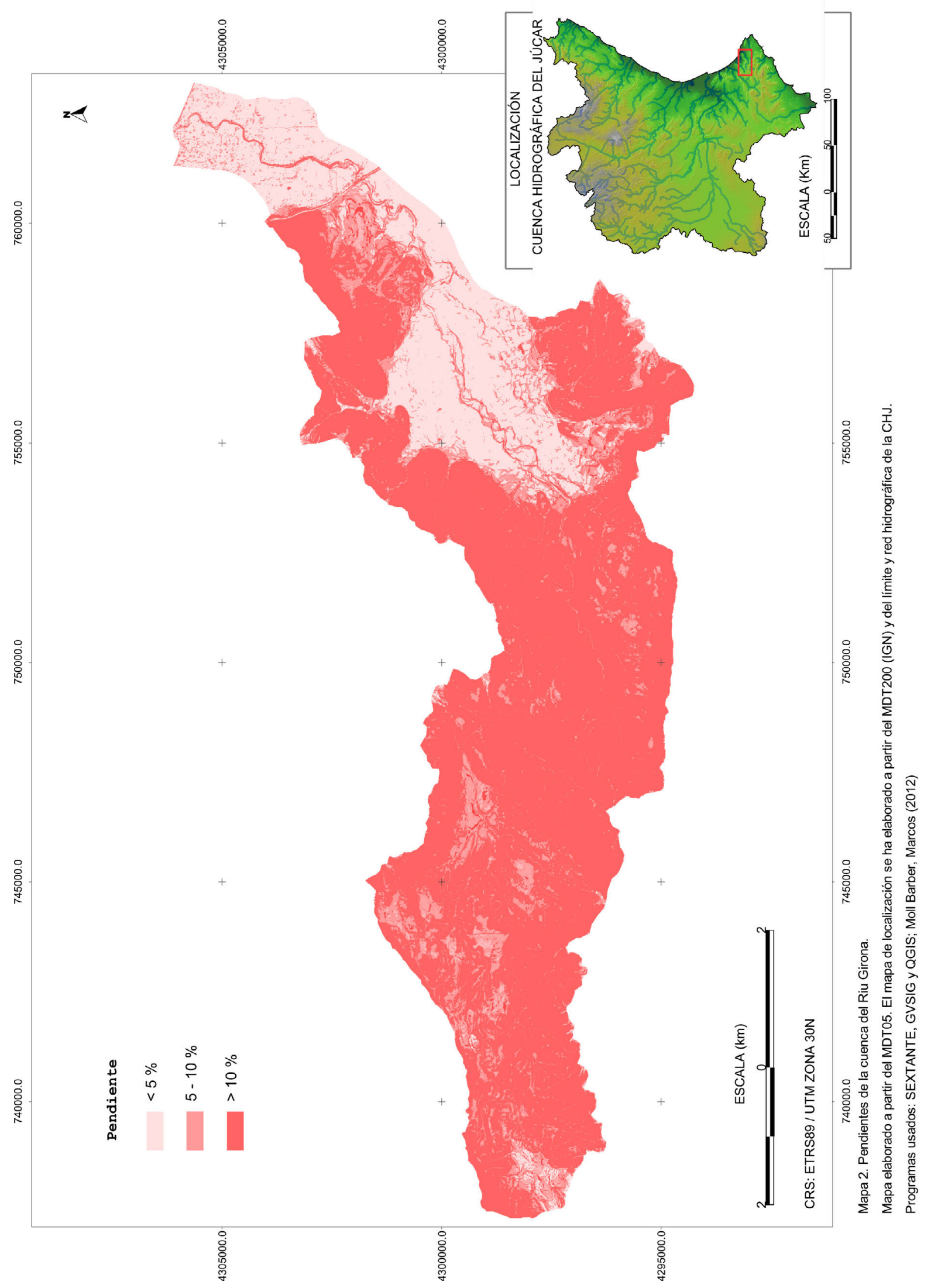




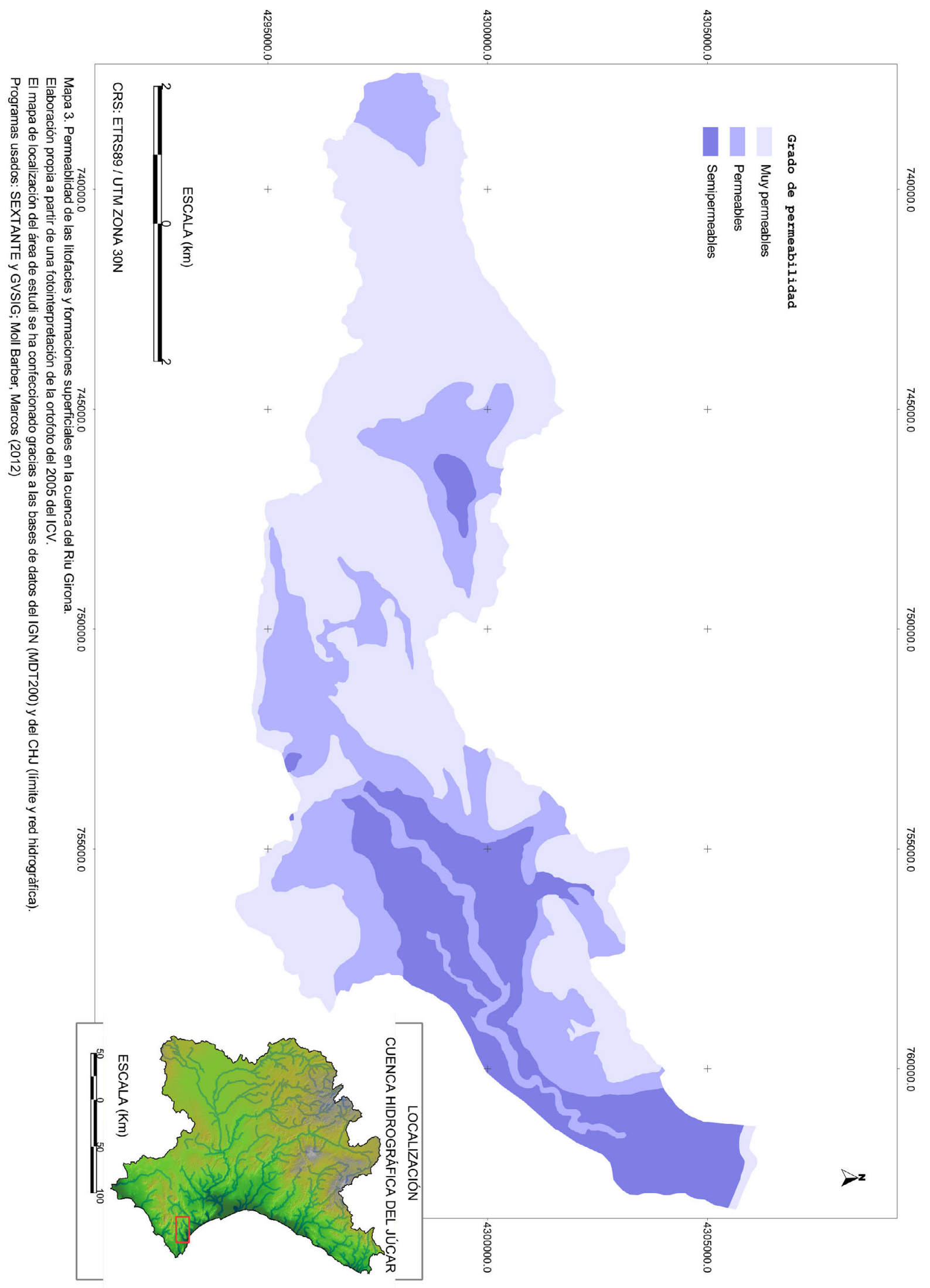


Una vez hecha la fotointerpretación se buscan los posibles errores cometidos en las geometrías, de la misma manera que se ha hecho con la capa de usos del suelo y vegetación, destacando que en ésta no se ha detectado ningún error geométrico.

\subsection{Fase de valoración e integración de las variables consideradas}

Antes de valorar las capas de información geográfica elaboradas anteriormente, es necesario establecer un coeficiente para combinar las variables que determinan la superficie de escorrentía $\left(V_{\text {se }}\right)$ y que éste se acerque al coeficiente de escorrentía $\left(C_{e}\right)$ de cada superficie. Para ello se ha optado por sumar las variables que intervienen en la producción de escorrentía y dividir el resultado entre el valor máximo posible de la combinación de los parámetros que se consideran.

$$
V_{s e}=\frac{C_{e}+m+a}{\operatorname{Max}}
$$

Donde " $V_{s e}$ " es el coeficiente para valorar las superficies de escorrentía; " $C_{e}$ " es el coeficiente de escorrentía según el uso del suelo y la cubierta vegetal; " $m$ " el valor incremental asignado a las pendientes; " $a$ " el valor asignado a las litofacies y las formaciones superficiales en relación al parámetro de infiltración; y "Max" es valor máximo resultante de la suma entre " $C_{e}$ ", " $m$ " y " $a$ ".

\subsubsection{Valoración de la capa de litofacies y formaciones superficiales}

Una vez establecido el coeficiente para valorar las superficies de escorrentía de la cuenca, se examinan las tablas de Marsh (1978), Benítez et al., (1980) y Prevert (1998) para cuantificar el incremento que experimenta el coeficiente de escorrentía en pasar de superficies permeables a semipermeables e impermeables. Considerando las superficies permeables como valor inicial, se comprueba que el coeficiente va incrementándose de manera constante; por tanto, ¿qué valor asignar al cambio de superficies permeables a impermeables o semipermeables? En la figura 4 se aclara el procedimiento llevado a cabo.

Figura 4. Ejemplos de los incrementos derivados del cambio de permeabilidad.

Cultivos agrícolas (pend. 5-10\%). Prevert (1998)

$$
\begin{aligned}
& 0,40=\text { perme. } \rightarrow 0,00 \\
& 0,66=\text { semip. } \rightarrow+0,16 \\
& 0,70=\text { imper. } \rightarrow+0,30
\end{aligned}
$$

Bosque (pend. 5-10\%). Benítez, et al., (1980)

$$
\begin{aligned}
& 0,25=\text { perme. } \rightarrow \quad 0,00 \\
& 0,45=\text { semip. } \rightarrow+0,20 \\
& 0,55=\text { imper. } \rightarrow+0,30
\end{aligned}
$$

Una vez contabilizados los incrementos que experimenta el $C_{e}$, según la permeabilidad, se calcula el valor medio de cada modelo y posteriormente la media de los tres (tabla 1). Cabe indicar que se cambian las denominaciones en muy permeables, permeables y semipermeables, reservando la denominación de superficies impermeables a aquellas compuestas por asfalto, hormigón, etc.

Tabla 1. Valor asignado al factor de permeabilidad (a) a partir de medias incrementales.

\begin{tabular}{|l|c|c|c|c|}
\hline $\begin{array}{c}\text { Litofacies y formaciones } \\
\text { superficiales }\end{array}$ & Marsh (1978) & Benítez, et al., (1980) & Prevert (1998) & Media a usar \\
\hline Muy permeable & 0,00 & 0,00 & 0,00 & 0,00 \\
\hline Permeable & 0,19 & 0,20 & 0,14 & 0,18 \\
\hline Semipermeable & 0,30 & 0,30 & 0,20 & 0,27 \\
\hline
\end{tabular}

\subsubsection{Valoración de la capa de pendientes}

Para valorar la pendiente se ha creído oportuno examinar las variaciones que experimentaba el coeficiente de escorrentía del método de Marsh (1978) al modificar la inclinación del terreno. En este caso, a diferencia del anterior, sólo se consideran los espacios que se encuentran en superficies permeables. 
De ese modo, considerando el valor de partida aquellos sectores con menor pendiente, se procede a cuantificar dicho cambio sin tener en cuenta valores acumulativos.

Figura 5. Incremento de la pendiente en usos del suelo desarrollados en superficies permeables.

\begin{tabular}{|c|c|c|}
\hline Bosque (sup. perm.) & Prado (sup. perm.) & Cultivos (sup. perm.) \\
$0,10=0-5 \% \rightarrow 0,00$ & $0,10=0-5 \% \rightarrow 0,00$ & $0,30=0-5 \% \rightarrow \quad 0,00$ \\
$0,15=5-10 \% \rightarrow+0,05$ & $0,16=5-10 \% \rightarrow+0,06$ & $0,40=5-10 \% \rightarrow+0,10$ \\
$0,30=10-30 \% \rightarrow+0,15$ & $0,22=10-30 \% \rightarrow+0,06$ & $0,52=10-30 \% \rightarrow+0,12$ \\
\hline
\end{tabular}

Tabla 2. Valor asignado a la pendiente $(m)$ a partir de la media incremental de Marsh (1978).

\begin{tabular}{|c|c|}
\hline Pendiente & Media a usar \\
\hline $0-5 \%$ & 0,00 \\
\hline $5-10 \%$ & 0,07 \\
\hline$>10 \%$ & 0,11 \\
\hline
\end{tabular}

\subsubsection{Valoración de los usos del suelo y cubierta vegetal}

Este proceso es fundamental para llevar a cabo la valoración de las superficies de escorrentía y, además, cabe señalar que es más complejo que las valoraciones anteriores. Por ello se ha creído oportuno hacer una revisión bibliográfica y extraer información cualitativa y cuantitativa de la repercusión de los usos del suelo y la cubierta vegetal en la escorrentía.

En primer lugar se valoran las superficies relacionadas con la actividad urbana. Para ello, se emplean los $C_{e}$ máximos que cuantifica Marsh (1978), siendo 0,75 en espacios urbanos concentrados y 0,80 en zona industrial ligera. A las urbanizaciones ex-novo se les otorga 0,60, a las áreas suburbanas 0,55 y 0,52 a las áreas deportivas, señalando que estos valores que se usan son mayores que los cuantificados por Marsh.

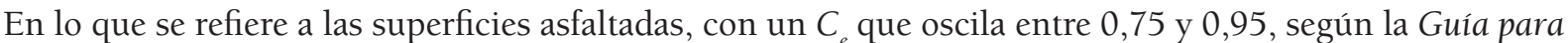
la Elaboración de Estudios del Medio Físico (Ministerio de Medio Ambiente, 2006), se opta por escoger el valor medio, 0,85; en la misma Guía aparece el $C_{e}$ de superficies de hormigón el cual fluctúa entre 0,80 y 0,95 , es por ello se que opta por usar el valor máximo para el lecho del río que está hormigonado. A los espacios no edificados 0,50, a los vertederos $0,45 \mathrm{y}$ a los jardines urbanos se les otorga un valor de 0,37.

En segundo lugar se plantea valorar las superficies relacionadas con la actividad agraria. Tomando como indicador el $C_{e}$ de cultivos desarrollados en superficies muy permeables y con poca pendiente del método de Marsh (1978), Benítez et al., (1980) y Prévert (1998), a los cultivos de regadío trabajados se les asigna un valor de 0,32 . Como contrapunto, los cultivos de regadío abandonados por el hecho de no labrarse se les otorgan un valor superior, de 0,39 . En lo que se refiere a las terrazas de cultivo, diferenciadas en trabajadas, primeros abandonos y abandono avanzado, a las primeras se les asigna 0,30 , al igual que en los cultivos de regadío; en cuanto a los cultivos abandonados con primeras colonizaciones vegetales se les asigna un valor de 0,35, mayor que el anterior; y a los cultivos con un abandono avanzado, 0,15 porque en ésta la cubierta vegetal aparece bien desarrollada. Este último valor se asigna porque las terrazas con un abandono avanzado y con una cubierta vegetal densa, generan una escorrentía insignificante (Cerdà, 1994). Considerando las balsas de regadío como recipiente de almacenamiento, se les otorga un valor de 0,00.

En tercer lugar se valoran las superficies relacionadas con espacios semi-naturales. En este sentido, en su día Molchanov (1960) demostró que a medida que aumentaba la densidad vegetal, el $C_{e}$ disminuía. Lo verificó experimentando con una superficie desprovista de vegetación y otra con un 60\% de cubierta vegetal; en la primera el fenómeno de escorrentía osciló entre 0,65 y 0,75 y en la segunda fluctuó sobre 0,08 (TRAGSA et al., 1998). Es por esta razón que las áreas consideradas como espacios forestales se les otorga un valor de 0,08 y a las áreas con maquia 0,10 . En lo que se refiere a los afloramientos rocosos, usando como indicador el estudio de Xiao-Yan et al., (2011), quienes en analizar el modelado cárstico de la Sierra de Gádor (SE de España) determinan que en zonas con afloramientos rocosos y calcáreos 
el $C_{e}$ fluctúa entre 0,59 y 0,80 , valores que vienen determinados por el grado de fisuras; a las zonas con afloramiento rocosos que a su vez se relacionan con incendios forestales pasados y con morfologías cársticas, se les concede un valor de 0,51 porque gran parte de su superficie se encuentra en pendientes superiores al $10 \%$, con un elevado grado de fisuras y con una cubierta vegetal regenerándose tras incendios y aprovechamientos forestales del pasado. En cuanto a la costa de acumulación se valora como superficie nula de escorrentía $(0,00)$ porque se trata de espacios donde la arena de la playa presenta una elevada tasa de infiltración. Por último, al lecho de la rambla se le asigna un valor de 0,60 para que aparezca destacado en el mapa final.

Tabla 3. Valoración del coeficiente de escorrentía según los usos del suelo y la vegetación $\left(C_{e}\right)$.

\begin{tabular}{|l|l|c|l|}
\hline 0,35 & Terrazas abandonadas (primeras colonizaciones) & 0,37 & Jardines urbanos \\
\hline 0,15 & Terrazas en abono avanzado (cubierta vegetal densa) & 0,50 & No edificado \\
\hline 0,30 & Terrazas en cultivo & 0,85 & Red de comunicaciones \\
\hline 0,32 & Cultivos regadío (trabajados) & 0,45 & Vertedero \\
0,36 & Cultivos regadío (abandonados) & 0,95 & Rambla (hormigonada) \\
\hline 0,00 & Balsa & 0,00 & Costa acumulación \\
\hline 0,75 & Urbano concentrado & 0,08 & Espacio forestal \\
\hline 0,60 & Urbanizaciones ex-novo & 0,10 & Maquia \\
\hline 0,55 & Zonas suburbanas & 0,51 & Afloramiento rocoso \\
\hline 0,52 & Áreas deportivas & 0,60 & Rambla \\
\hline 0,80 & Zona industrial & & \\
\hline
\end{tabular}

\subsubsection{Integración de las variables}

Finalmente se procede a realizar el geoproceso de intersección entre las tres capas consideradas. Una vez combinadas a la capa final se le modifica la tabla, creando una nueva columna en donde se introduce la ecuación anterior (figura 4) y de ese modo se obtiene una capa de información geográfica en la que consta la valoración de las superficies de escorrentía.

\subsection{Fase cartográfica de verificación}

Con el objetivo de verificar el resultado de lo expuesto en este estudio aplicado se han comprobado si los valores que resultan de la valoración de la escorrentía concuerdan con los coeficientes de escorrentía explicados en el segundo apartado. Además de ello se han realizado salidas de campo para contrastar los resultados obtenidos.

\section{RESULTADOS, DISCUSIÓN Y APLICABILIDAD}

El estudio revela que mediante las nuevas tecnologías ligadas a los SIG se puede cartografiar y llevar a cabo una valoración de las superficies de escorrentía de una cuenca. A grandes rasgos, el resultado que se obtiene de la valoración y combinación de las variables manejadas, pendiente, litofacies y formaciones superficiales y usos del suelo y cubierta vegetal, se aproxima bastante a la realidad de la cuenca de estudio. No obstante, el método usado debería perfeccionarse y, en la medida de lo posible, sería conveniente experimentar con coeficientes de escorrentía cuantificados en la propia cuenca de estudio para poder confeccionar mejor el método de valoración de las variables. Además de ello, sería interesante que en la valoración se tuviera en cuenta el factor de insolación en umbrías y solanas, ya que en las umbrías el coeficiente de escorrentía debería ser mayor por el hecho de que los suelos se mantienen más húmedos, auque habría que considerar que en estos sectores suele hallarse un mayor recubrimiento vegetal. 


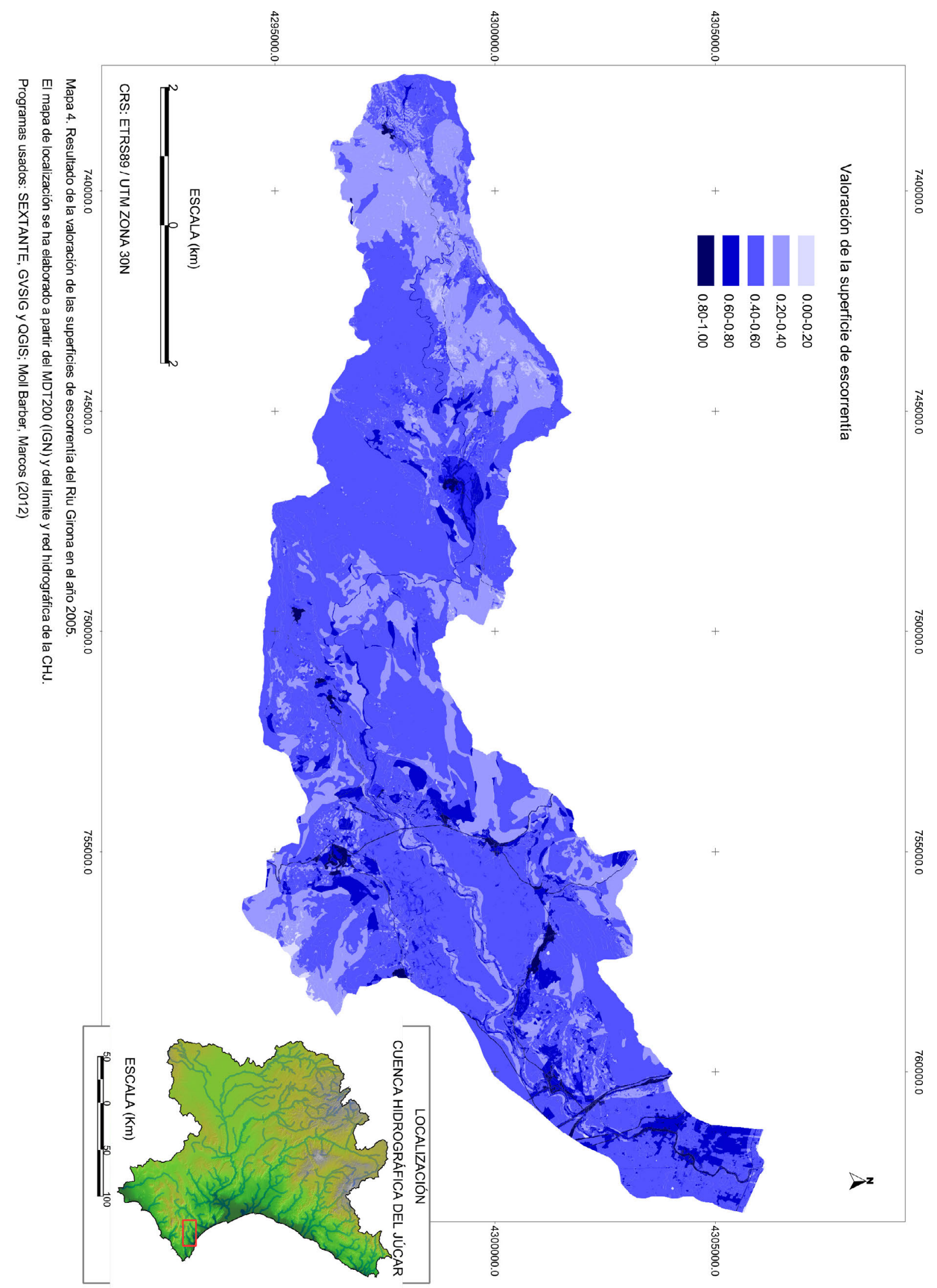


Del resultado final cabe destacar que el 67,05\% de la superficie de escorrentía obtiene valores entre 0,40 y 0,60 , y éstos se corresponden fundamentalmente con afloramientos rocosos, cultivos de regadío y terrazas cultivadas, entre otros. Un porcentaje menor, de 23,33\%, se corresponde con superficies de escorrentía con unos valores entre 0,20 y 0,40, éstos son espacios con maquia, masa arbórea densa, cultivos con abandonos avanzados, etc. El 7,71\% de la superficie de escorrentía obtiene valores entre 0,60 y 0,80 , correspondiéndose principalmente con espacios donde se asientan nuevas urbanizaciones, entre otros. El 1,76\% de la superficie de escorrentía se engloba entre valores de 0,80 y 1,00, éstos se relacionan con espacios urbanos consolidados, vías de comunicaciones, rambla hormigonada, etc. El 0,23\% de la superficie de escorrentía obtienen valores de entre 0,00 y 0,20 , resultando ser aquéllos espacios con una cubierta vegetal densa y con poca pendiente.

A modo de ejemplo de la aplicabilidad del mapa final, y sin entrar en detalles (tiempos de concentración, etc.), se ha calculado el caudal de salida de la cuenca de estudio usando intensidades de lluvia homogéneas en toda la cuenca (figura 6). Las intensidades que se han manejado han sido de $60 \mathrm{~mm} / \mathrm{h}$ y de $120 \mathrm{~mm} / \mathrm{h}$, este último valor se corresponde a las intensidades de lluvia registradas en el observatorio de Gallinera, cercano a la zona de estudio, entre las 10'45 y las 11'00 el 12 de octubre de 2007 (Segura, 2009).

Tabla 4. Cálculo del caudal $\left(\mathrm{m}^{3} / \mathrm{s}\right)$ de salida según la intensidad de la lluvia y por tramos*.

\begin{tabular}{|c|l|c|c|c|c|}
\hline \multirow{2}{*}{ Zona } & \multirow{2}{*}{ Pueblos } & \multicolumn{2}{c|}{$\begin{array}{c}\text { Caudal por tramos según intensidades } \\
\text { de lluvia }\end{array}$} & \multicolumn{2}{c|}{$\begin{array}{c}\text { Caudal acumulado según intensidad } \\
\text { de la lluvia }\end{array}$} \\
\cline { 3 - 6 } & & $60 \mathrm{~mm} / \mathrm{h}$ & $120 \mathrm{~mm} / \mathrm{h}$ & $60 \mathrm{~mm} / \mathrm{h}$ & $120 \mathrm{~mm} / \mathrm{h}$ \\
\hline 1 & Vall d'Alcalà y Vall d'Ebo & 448,5123 & 897,0246 & 448,5123 & 897,0246 \\
\hline 2 & La Vall de Laguar & 349,6999 & 699,3998 & 798,2122 & 1596,4244 \\
\hline 3 & Orba y La Rectoria & 459,2085 & 918,4170 & 1257,4207 & 2514,8414 \\
\hline 4 & Beniarbeig, El Verger y Els Poblets & 206,6705 & 413,3411 & 1464,0912 & 2928,1825 \\
\hline
\end{tabular}

* Fórmula aplicada a cada una de las superficies de escorrentía obtenidas en la valoración ${ }^{3}$

$$
Q=\frac{C \cdot I \cdot A}{3.000 .000}
$$

Donde:

$Q$ = Caudal punta correspondiente a un período de retorno dado en $\mathrm{m}^{3} / \mathrm{s}$.

$I=$ Intensidad de la lluvia en $\mathrm{mm} / \mathrm{h}$.

$A=$ Superficie de escorrentía dada en $\mathrm{m}^{2}$.

$C=$ Coeficiente de escorrentía obtenido en la valoración.

Figura 6. Caudal de salida acumulado obtenido ante intensidades pluviométricas de $120 \mathrm{~mm} / \mathrm{h}$.

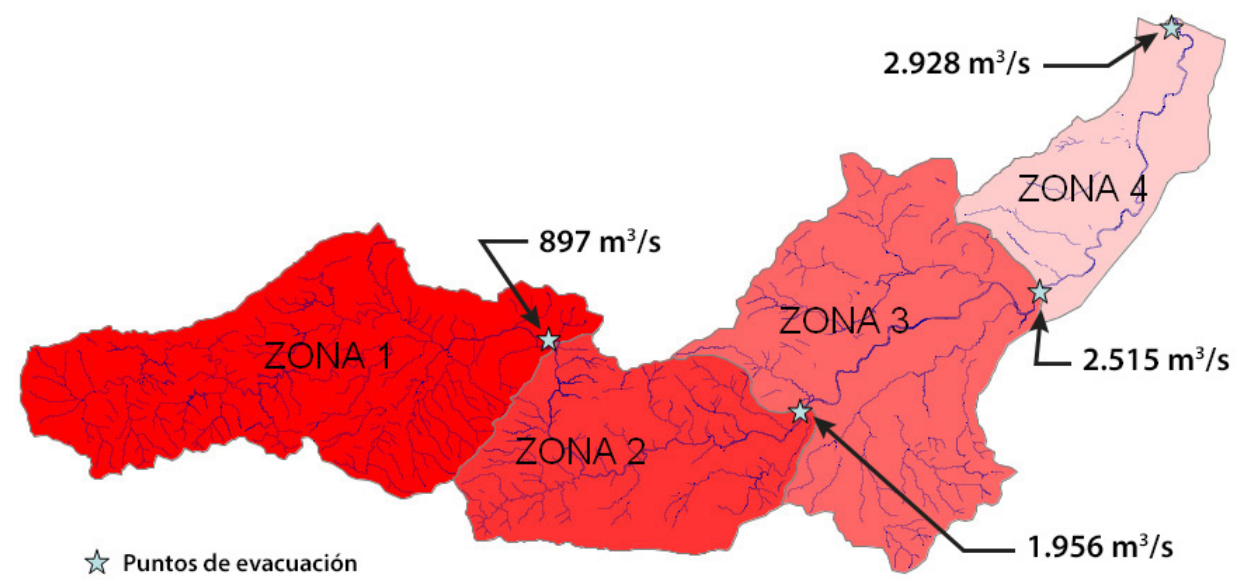

3 La fórmula se ha extraído de MOPU (1987) y se ha modificado puntualmente como adaptación a la información manejada. 


\section{BIBLIOGRAFÍA}

ALlARD, A., NILSSON, B., PRAMBORG, K., STAHL, G. and SUNDQUIST, S. (2003): Manual for aerial photo interpretation in the national inventoy of landscapes in Sweden. NILS, Deparment of Forest Resource Management and Gemomatics, Sweden, 80 p. URL: < http://www.slu.se >

ANGElone, S., GARIBAY, M.T. y CAUHAPÉ CASAUX, M. (2006): "Permeabilidad de suelos". Universidad Nacional de Rosario. 39 p.

ARCENEGUI, V. (2011): "El foc sobre el sòl, els canvis en la superfície després d'un incendi", Mètode "Quan es crema el bosc", núm 70, pp. 79-81. Universitat de València, València.

BENÍTEZ, C., ARIAS y W., QUIROZ, J. (1980): Manual de conservación de suelos y aguas. Ministerio de Agricultura y Alimentación. Perú.

CAROLL, D. M., EVANS, R. and BENDELOWS, V.C. (1977): Air photo-interpretation for soil mapping., England, 85 p.

CERDÀ, A. y BODÍ, M.B. (2006): "Erosión hídrica en suelos afectados por incendios forestales", en MATAIX SOLERA, J. (ed.) (2006): Incendios forestales, suelos y erosión hídrica, pp. 71-118

CERDÀ I BOLICHES, A. (1994): "Arroyada superficial en terrazas de cultivo abandonadas: el caso del País Valencià” a Quaderns de Geografia no 56, pp. 135-154. València.

CONACHER, A. and SALA, M. (1998): Land degradation in mediterranean environments of the world. Ed. John Wiley \& Sons.

GARCÍA RODRÍGUEZ, J. L. and GIMÉNEZ SUÁREZ, C. (2010): “Comparison of mathematical algorithms for determining the slope angle in GIS environment”, Aqua-LAC, vol. 2, n 2. pp. 78-82. URL: < www. unesco.org >

GARCÍA-RUIZ, J. M., RUIZ-FLAÑO, P., LASANTA, T., MONTSERRAT, G., MARTÍNEZ-RICA, J.P. and PARDINI, G. (1991): "Erosion in abandoned field, what is the problem?". In SALA, M., RUBIO, J. L. i GARCÍA-TRUI, J. M. (Eds.): Soil Erosion Studies in Spain, pp. 97-108

GARCÍA RUIZ, J.M. (1996): "Marginación de tierra y erosión en áreas de montaña". En LASANTA MARTÍNEZ, T. y GARCÍA RUIZ, J.M. (eds.) (1996): Erosión y recuperación de tierras en áreas marginales. Instituto de Estudios Riojanos. SEG. Logroño. pp. 33-50.

GIL OlCINA, A. (Dir.) (1986): Inundaciones en la ciudad y Término de Alicante. Universitat d'Alacant Ajuntament d'Alacant, $179 \mathrm{p}$.

GIOVANNINI, G. and LUCCHESI, S. (1997): "Modifications induced in soil physico-chemical parameters by experimental fires at different intensities". Soil Science, núm. 162-7, pp. 479 - 486.

GONZÁLEZ, A. y MOÑINO, M. (2000): "Foto-interpretación geomorfológica aplicada al estudio de la evolución del relieve", Enseñanza de las Ciencias de la Tierra, pp. 23-31.

HENRÍQUEZ, C. AZÓCAR, G. y AGUAYO, M. (2006): "Cambio de usos del suelo y escorrentía superficial: aplicación de un modelo de simulación espacial en Los Ángeles, VIII Región del Biobío, Chile”, Revista de Geografía Norte Grande, núm. 36, Santiago. pp. 61-74. URL: < http://www.scielo.cl >

HERRERO TEJEDOR, T.R. y PÉREZ MARTÍN, E. (2002): “Actualización topogràfica y cartogràfica mediante técnicas GPS y SIG del Monte Público, aguas vertientes el Espinar (Segovia)”, XIV Congreso Internacional de Ingeniería Gràfica, Santander, España, 10 p.

HORTON, R. E.:

- (1933): "The role of infiltration in the hydrologic cycle", Trans. 14t Ann. Meeting Am. Gephys. Union, pp. $446-460$.

- (1939): "Analysis of runoff-pot experiments with varying infiltration capacity", Trans. Am. Geo. Union, pp. $693-694$. 
IBÁÑEZ ASENSIO, S., MORENO RAMÓN, H. y GISBERT BLANQUER, J. M. (2007): "Métodos para la determinación del coeficiente de escorrentía". Universitat Politècnica de València. 7 p. URL: < http:// riunet.upv.es >

KOSTIAKOV, A.N. (1932): "On the dynamics of the coefficient of water percolation in soils and the necessity for studying it from a dynamic riew for purposes of amelsoration", Trans. 6th Com. Int. Soil Sc., Russian Part A, pp.17-21.

LEMUS, M. y NAVARRO, G. (2003) Manual para el desarrollo de obras de conservación del suelo. Corporación Forestal Nacional. San Fernando (Chile)

LLOVET LÓPEZ, J. i VALLEJO CALZADA, V. R. (2011): "Foc, pluges i resposta hidrològica del sòl a les muntanyes d'Alacant", Treballs de la Societat Catalana de Geografia, núm. 71-72, pp. 35 - 47. URL: < http://publicacions.iec.cat >

MARSH, W. M. (1978): Environmental analysis for land use and site planning. McGraw-Hill, New York.

MARTÍN VIDE, J. (2006): "La importancia de los estudios históricos en el análisis de los riesgos climáticos", en AYALA CARCEDO, F. J. et al., (2006): Riesgos naturales y desarrollo sostenible: impacto, predicción y mitigación. Instituto Geológico y Minero de España, Madrid, pp. 159-166.

MAS LlORENÇ, M. A. (1993): Atlas de la Marina Alta. Institut Geogràfic d'Alacant. Ed. CAM, Fundación Cultural.

MATAIX SOLERA, J. y GUERRERO, C. (2006): "Efectos de los incendios forestales en las propiedades edáficas” en MATAIX SOLERA, J. (ed.) (2006): Incendios forestales, suelos y erosión hídrica, pp. 5 - 40.

MATEU, J. F., PORTUGÉS i GÓMEZ G, GARCIA y F., FERRI, M. (2010): La primera confederación hidrográfica del Júcar (1934-.1942). Confederacio Hidrográfica del Xúquer, València, 155 p.

MINISTERIO DE MEDIOAMBIENTE (2006): Guía para la elaboración de estudios del medio físico. Madrid, $917 \mathrm{p}$.

MIRAJKAR, M. A. and SRINJVASAN, T. R. (1975): "Landsat photo-interpretation for preparation of small scala soil maps through a multistage approach", Photonirvachak Journal of the Indian Society of remote sensig, Vol. III, núm. 2, pp. 87-92.

MOLINA, M.J. and SANROQUE, P. (1996): "Impact of forest fires on desertification processes: a review in relation to soil erodibility". In: RUBIO, J. L., CALVO, A. (eds.): Soil degradation and desertification in Mediterranean environments. Logronyo: Geoforma Ediciones, pp. 145-163.

MOPU (1987): "Cálculo hidrometeorológico de caudales máximos en pequeñas cuencas naturales", Tecnología. Madrid, 49 p. URL: < http://www.fomento.gob.es>

MUSGRAVE, G. W. and HOLTAN, H. N. (1964): Infiltraron, Section 12. Ven Te Chow (ed.): Handbook of applied hydrology, IV. Graw-Hill. New York.

NEARY, D.G., KLOPATEK, C.C., DE BANO, L.F. and FFOLliOTT, P.F. (1999): "Fire effects on belowground sustainability: a review and synthesis". Forest Ecology and Management, núm. 122, pp. 51-71.

OLCINACANTOS,J.,HERNÁNDEZHERNÁNDEZ, M., RICOAMORÓS,A. M.andMARTÍNEZIBARRA, E. (2010): "Increased risk of flooding on the coast of Alicante (Region of Valencia, Spain)" a Natural Hazards and Earth System Sciences, núm 10.6 p. URL:< www.nat-hazards-earth-syst-sci.net >

PADILLA BLANCO, A. (1998): Colonización vegetal en campos de cultivo abandonados en la provincia de Alicante. Universitat d'Alacant. 365 p.

PHILIP, J. R. (1957): "The theory of infiltration: 4. Sorptivity and algebraic infiltration equations", Soil Sc. 84 , pp. 257- 264.

SHAKESBY, R.A. and DOERR, S.H. (2006): "Wildfire as a hydrological and geomorphological agent". Earth-Science Reviews, núm. 74, 3-4, pp. 269-307. 
SMITH, R.E. (1972): "The infiltration envelope: results from a theoretical infiltrometer". Journa of Hydrology, 17, pp. 1-21.

SOIL SURVEY STAFF (1951): Soil survey manual. USDA, Handbook 18, Washington.

TÉMEZ, J.R. (1978): Cálculo hidrometeorológico de caudales máximos en pequeñas cuencas naturales. MOPU Dirección General de Carreteras, 113 p.

TRAGSA, TRAGSATEC (1998): Restauración hidrológica forestal de cuencas y control de la erosión. Ministerio del Medio Ambiente. Ediciones mundi prensa Madrid.

TRILLA I ARRUFAT, J., MAS I PLA, J. i PALLÍ I BUXÓ, Ll. (1985): "Imposicions estructurals en el drenatge de la conca del riu Onyar (Girona)", Scientia gerundensis, núm 11. pp 103-118. URL: < www. $\underline{\text { raco.cat }>}$

ÚBEDA, X.:

- (1999): "Canvis en la capacitat d'infiltració d'un sòl després d'un incendi forestal al massís de Cadiretes. Importància de la intensitat del foc en les taxes d'infiltració". Scientia gerundensis, núm 24, pp. 41-51.

- (2000): "Els incendis forestals com a causants de l'increment d'erosió del sòl". Butlletí Instituts Catalans de la Història Natural. núm 68, pp. 5-14. URL: < www.revistes.iec.cat >

VÉLEZ MUÑOZ, R. y MONTIEL MOLINA, C. (2003): "La problemática de monte mediterráneo". Investigaciones Geográficas, núm 31, pp. 121-137. Universitat d'Alacant, Alacant. URL: < http://rua. $\underline{\text { ua.es }>}$

XIAO-YAN, L., CONTRERAS, S., SOLÉ.BENET, A., CANTÓ, Y., DOMINGO, F., LÁZARO, R., LIN, H., VAN WESEMAEL, B. and PUIGDEFÁBREGAS, J. (2011): "Controls of infiltrationrunoff processes in Mediterranean karst rangelands in SE Spain". Catena, Vol. 86, pp. 98 -109. URL: < http://www.sciencedirect.com > 